Pacific Journal of Mathematics

PRODUCT INTEGRAL REPRESENTATION OF TIME
DEPENDENT NONLINEAR EVOLUTION EQUATIONS IN

GLenN Francis Web 


\title{
PRODUCT INTEGRAL REPRESENTATION OF TIME DEPENDENT NONLINEAR EVOLUTION EQUATIONS IN BANACH SPACES
}

\author{
G. F. WeBB
}

The object of this paper is to use the method of product integration to treat the time dependent evolution equation $u^{\prime}(t)=A(t)(u(t)), t \geqq 0$, where $u$ is a function from $[0, \infty)$ to a Banach space $S$ and $A$ is a function from $[0, \infty)$ to the set of mappings (possibly nonlinear) on $S$. The basic requirements made on $A$ are that for each $t \geqq 0 A(t)$ is the infinitesimal generator of a semi-group of nonlinear nonexpansive transformations on $S$ and a continuity condition on $A(t)$ as a function of $t$.

The product integration method has been used by T. Kato in [5] to treat evolution equations in which $A(t)$ is the infinitesimal generator of a semi-group of linear contraction operators. In [6] Kato treats the nonlinear evolution equation in which $A(t)$ is $m$-monotone and the Banach space $S$ is uniformly convex. For other investigations of nonlinear evolution equations one should see P. Sobolevski [9], F. Browder [1], J. Neuberger [8], and J. Dorroh [3].

1. Definitions and theorems. In this section definitions and theorems will be stated. For examples satisfying the definitions and theorems below, one should see $\S 4$. Let $S$ denote a real Banach space.

Definition 1.1. The function $T$ from $[0, \infty)$ to the set of mappings (possibly nonlinear) on $S$ will be said to be a $\mathscr{C}$-semi-groups of mappings on $S$ provided that the following are true:

(1) $T(x+y)=T(x) T(y)$ for $x, y \geqq 0$.

(2) $T(x)$ is nonexpansive for $x \geqq 0$.

(3) If $p \in S$ and $g_{p}(x)$ is defined as $T(x) p$ for $x \geqq 0$ then $g_{p}$ is continuous and $g_{p}(0)=p$.

(4) The infinitesimal generator $A$ of $T$ is defined on a dense subset $D_{A}$ of $S$ (i.e., if $p \in D_{A} g_{p}^{\prime+}(0)$ exists and $A p=g_{p}^{\prime+}(0)$ ) and if $p \in D_{A} g_{p}^{\prime+}(x)=A g_{p}(x)$ for $x \geqq 0, g_{p}(x)=p+\int_{0}^{x} A g_{p}(u) d u$ for $x \geqq 0, g_{p}^{\prime+}$ is continuous from the right on $[0, \infty)$, and $\left\|g_{p}^{\prime+}\right\|$ is nonincreasing on $[0, \infty)$.

Definition 1.2. The mapping $A$ from a subset of $S$ to $S$ will be said to be a $\mathscr{C}$-mapping on $S$ provided that the following are true:

(1) The domain $D_{A}$ of $A$ is dense in $S$. 
(2) $A$ is monotone on $S$, i.e., if $\varepsilon>0$ and

$$
p, q \in D_{A}\|(I-\varepsilon A) p-(I-\varepsilon A) q\| \geqq\|p-q\| \text {. }
$$

(3) $A$ is $m$-monotone on $S$, i.e. $A$ is monotone on $S$ and if $\varepsilon>0$ then Range $(I-\varepsilon A)=S$.

(4) $A$ is the infinitesimal generator of a $\mathscr{C}$-semi-group of mappings on $S$.

DEFINITION 1.3. Let each of $m$ and $n$ be a nonnegative integer and for each integer $i$ in $[m, n]$ let $K_{i}$ be a mapping from $S$ to $S$. If $m>n$ define $\prod_{i=m}^{n} K_{i}=I$. If $m \leqq n$ define $\prod_{i=m}^{m} K_{i}=K_{m}$ and if $m+1 \leqq j \leqq n$ define $\prod_{i=m}^{j} K_{i}=K_{j} \prod_{i=m}^{j-1} K_{i}$. Define $\prod_{n}^{i=m} K_{i}=\prod_{i=m}^{n} K_{n+m-i}$. If each of $a$ and $b$ is a nonnegative number then a chain $\left\{s_{i}\right\}_{\imath=0}^{2 m}$ from $a$ to $b$ is a nondecreasing or nonincreasing number-sequence such that $s_{0}=a$ and $s_{2 m}=b$. The norm of $\left\{s_{i}\right\}_{i=0}^{2 m}$ is $\max \left\{\left|s_{2 i}-s_{2 i-2}\right| \mid i \in[1, m]\right\}$.

Definition 1.4. Let $F$ be a function from $[0, \infty) \times[0, \infty)$ to the set of mappings on $S$. Suppose that $p \in S, a, b \geqq 0$, and $u$ is a point in $S$ such that if $\varepsilon>0$ there exists a chain $\left\{s_{i}\right\}_{i=0}^{2 m}$ from $a$ to $b$ such that if $\left\{t_{i}\right\}_{i=0}^{2 n}$ is a refinement of $\left\{s_{i}\right\}_{i=0}^{2 m}$ then

$$
\left\|u-\prod_{i=1}^{n} F\left(t_{2 i-1},\left|t_{2 i}-t_{2 i-2}\right|\right) p\right\|<\varepsilon .
$$

Then $u$ is said to be the product integral of $F$ from $a$ to $b$ with respect to $p$ and is denoted by $\prod_{a}^{b} F(I, d I) p$.

REMARK 1.1. Let $A$ be a $\mathscr{C}$-mapping on $S$ and define the function $F$ from $[0, \infty) \times[0, \infty)$ to the set of mappings on $S$ by $F(u, v)=$ $(I-v A)^{-1}$ for $u, v \geqq 0$ (Note that $(I-v A)^{-1}$ exists and has domain $S$ by virtue of the $m$-monotonicity of $A$ ). The following result in [10] will be used in the theorems below:

If $A$ is a $\mathscr{C}$-mapping on $S, T$ is the $\mathscr{C}$-semi-group generated by $A$, and $F$ is defined as above, then for $p \in S$ and $x \geqq 0 T(x) p=$ $\Pi_{0}^{x} F(I, d I) p$.

In this case let $T(x)$ be denoted by $\exp (x A)$ for $x \geqq 0$.

Let $A$ be a function from $[0, \infty)$ to the set of mappings on $S$ such that the following are true:

( I ) For each $t \geqq 0 A(t)$ is a $\mathscr{C}$-mapping on $S$

(II) There is a dense subset $D$ of $S$ such that if $t \geqq 0$ the domain of $A(t)$ is $D$

(III) $A$ is continuous in the following sense: If $a, b \geqq 0, M$ is a bounded subset of $D$, and $\varepsilon>0$, there exists $\delta>0$ such that if $u, v \in[a, b]$ and $|u-v|<\delta$ then $\|A(u) z-A(v) z\|<\varepsilon$ for each $z \in M$. 
THEOREM 1. Let A satisfy conditions (I), (II) and (III). If $p \in S$ and $a, b \geqq 0$ the following are true:

(1) If $T(u, v)=\exp (v A(u))$ for $u, v \geqq 0$, then $\prod_{a}^{b} T(I, d I) p$ exists.

(2) If $L(u, v)=(I-v A(u))^{-1}$ for $u, v \geqq 0$, then $\Pi_{a}^{b} L(I, d I) p$ exists and $\prod_{a}^{b} L(I, d I) p=\prod_{a}^{b} T(L, d I) p$.

THEOREM 2. Let A satisfy conditions (I), (II) and (III) and define $U(b, a) p=\prod_{a}^{b} T(I, d I) p$ for $p \in S$ and $a, b \geqq 0$. The following are true:

(1) $U(b, a)$ is nonexpansive for $a, b \geqq 0$.

(2) $U(b, c) U(c, a)=U(b, a)$ for $a, b \geqq 0$ and $c \in[a, b]$ and $U(a, a)=$ I for $a \geqq 0$.

(3) If $p \in S$ and $a \geqq 0$ then $U(a, t) p$ is continuous in $t$

(4) If $p \in S, 0 \leqq a \leqq t$, and $U(t, a) p \in D$, then $\partial^{+} U(t, a) p / \partial t=$ $A(t) U(t, a) p$ and if $p \in S, 0<s \leqq b$, and $U(s, b) p \in D$, then

$$
\partial^{-} U(s, b) p / \partial s=-A(s) U(s, b) p .
$$

2. Product integral representations. In this section, Theorems 1 and 2 will be proved. Before proving part (1) of Theorem 1 three lemmas will be proved each under the hypothesis of Theorem 1.

Lemma 1.1. If $p \in D, a, b \geqq 0$, and $\left\{s_{i}\right\}_{i=0}^{2 m}$ is a chain from a to $b$ then

$$
\left\|\prod_{i=1}^{m} T\left(s_{2 i-1},\left|s_{2 i}-s_{2 i-2}\right|\right) p-p\right\| \leqq \sum_{i=1}^{m}\left|s_{2 i}-s_{2 i-2}\right|\left\|A\left(s_{2 i-1}\right) p\right\| .
$$

Proof.

$$
\begin{aligned}
& \left\|\prod_{i=1}^{m} T\left(s_{2 i-1},\left|s_{2 i}-s_{2 i-2}\right|\right) p-p\right\| \\
& \quad \leqq \sum_{i=1}^{m}\left\|\prod_{j=2}^{m} T\left(s_{2 j-1},\left|s_{2 j}-s_{2 j-2}\right|\right) p-\prod_{j=i+1}^{m} T\left({ }_{2 j-1},\left|s_{2 j}-s_{2 j-2}\right|\right) p\right\| \\
& \quad \leqq \sum_{i=1}^{m}\left\|T\left(s_{2 i-1},\left|s_{2 i}-s_{2 i-2}\right|\right) p-p\right\| \\
& \quad=\sum_{i=1}^{m}\left\|\int_{0}^{\mid s_{2 i-s_{2 i-2} \mid}} A\left(s_{2 i-1}\right) T\left(s_{2 i-1}, t\right) p d t\right\| \\
& \quad \leqq \sum_{i=1}^{m}\left|s_{2 i}-s_{2 i-2}\right| \cdot\left\|A\left(s_{2 i-1}\right) p\right\|
\end{aligned}
$$

LEMMA 1.2. If $p \in D, a, b \geqq 0,\left\{s_{i}\right\}_{i=0}^{2 m}$ is a chain from $a$ to $b$, and $\left\{s_{i}^{\prime}\right\}_{i=1}^{m}$ is a sequence in $[a, b]$, then

$$
\left\|\prod_{i=1}^{m} L\left(s_{i}^{\prime},\left|s_{2 i}-s_{2 i-2}\right|\right) p-p\right\| \leqq \sum_{i=1}^{m}\left|s_{2 i}-s_{2 i-2}\right|\left\|A\left(s_{i}^{\prime}\right) p\right\| .
$$

Proof. 


$$
\begin{aligned}
&\left\|\prod_{i=1}^{m} L\left(s_{i}^{\prime},\left|s_{2 i}-s_{2 i-2}\right|\right) p-p\right\| \\
& \leqq \sum_{i=1}^{m}\left\|\prod_{j=i}^{m} L\left(s_{j}^{\prime},\left|s_{2 j}-s_{2 j-2}\right|\right) p-\prod_{j=i+1}^{m} L\left(s_{j}^{\prime},\left|s_{2 j}-s_{2 j-2}\right|\right) p\right\| \\
& \leqq \sum_{i=1}^{m}\left\|L\left(s_{i}^{\prime},\left|s_{2 i}-s_{2 i-2}\right|\right) p-p\right\| \\
&= \sum_{i=1}^{m} \| L\left(s_{i}^{\prime},\left|s_{2 i}-s_{2 i-2}\right|\right) p \\
&-L\left(s_{i}^{\prime},\left|s_{2 i}-s_{2 i-2}\right|\right)\left(I-\left|s_{2 i}-s_{2 i-2}\right| A\left(s_{i}^{\prime}\right)\right) p \| \\
& \leqq \sum_{i=1}^{m}\left|s_{2 i}-s_{2 i-2}\right| \cdot\left\|A\left(s_{i}^{\prime}\right) p\right\|
\end{aligned}
$$

LEMMA 1.3. If $M$ is a bounded subset of $D, a, b \geqq 0, \gamma>0$, and $\varepsilon>0$, there exists $\delta>0$ such that if $u, v \in[a, b],|u-v|<\delta, 0 \leqq x<\gamma$, and $z \in M$, then $\|T(u, x) z-T(v, x) z\| \leqq x \cdot \varepsilon$.

Proof. Let $M^{\prime}=\left\{\prod_{i=1}^{m} L\left(v, s_{2 i}-s_{2 i-2}\right) z \mid z \in M, v \in[a, b], 0 \leqq x<\gamma\right.$, and $\left\{s_{i}\right\}_{i=0}^{2 m}$ is a chain from 0 to $\left.x\right\}$. Let $z_{0} \in M$, let $z \in M$, let $v \in[a, b]$, let $0 \leqq x<\gamma$, and let $\left\{s_{i}\right\}_{i=0}^{2 m}$ be a chain from 0 to $x$. Then,

$$
\left\|\prod_{i=1}^{m} L\left(v, s_{2 i}-s_{2 i-2}\right) z-\prod_{i=1}^{m} L\left(v, s_{2 i}-s_{2 i-2}\right) z_{0}\right\| \leqq\left\|z-z_{0}\right\| .
$$

Further, by Lemma 1.2,

$$
\left\|\prod_{i=1}^{m} L\left(v, s_{2 i}-s_{2 i-i}\right) z_{0}-z_{0}\right\| \leqq x \cdot \max _{u \in \mid 0, x\rceil}\left\|A(u) z_{0}\right\| .
$$

Then, $\left\|\prod_{\imath=1}^{m} L\left(v, s_{2 i}-s_{2 i-2}\right) z\right\| \leqq\left\|z-z_{0}\right\|+\left\|z_{0}\right\|+x \cdot \max _{u \in[0, \gamma]}\left\|A(u) z_{0}\right\|$ and so $M^{\prime}$ is bounded. There exists $\delta>0$ such that if $u, v \in[a, b]$, $|u-v|<\delta$, and $z \in M^{\prime}$, then $\|A(u) z-A(v) z\|<\varepsilon$. Then if $0 \leqq x<\gamma$, $z \in M,\left\{s_{i}\right\}_{i=0}^{2 m}$ is a chain from 0 to $x, u, v \in[a, b]$, and $|u-v|<\delta$,

$$
\begin{aligned}
&\left\|\prod_{i=1}^{m} L\left(u, s_{2 i}-s_{2 i-2}\right) z-\prod_{i=1}^{m} L\left(v, s_{2 i}-s_{2 i-2}\right) z\right\| \\
& \leqq \sum_{i=1}^{m} \| \prod_{j=i}^{m} L\left(u, s_{2 j}-s_{2 j-2}\right) \prod_{k=1}^{i-1} L\left(v, s_{2 k}-s_{2 k-2}\right) z \\
& \quad-\prod_{j=-211}^{m} L\left(u, s_{2 j}-s_{2 j-2}\right) \prod_{k=1}^{i} L\left(v, s_{2 k}-s_{2 k-2}\right) z \| \\
& \leqq \sum_{i=1}^{m} \| L\left(u, s_{2 i}-s_{2 i-2}\right) \prod_{k=1}^{i-1} L\left(v, s_{2 k}-s_{2 k-2}\right) z \\
& \quad-\prod_{k=1}^{i} L\left(v, s_{2 k}-s_{2 k-2}\right) z \| \\
& \leqq \sum_{i=1}^{m} \| \prod_{k=1}^{i-1} L\left(v, s_{2 k}-s_{2 k-2}\right) z \\
& \quad-\left(I-\left(s_{2 i}-s_{2 i-2}\right) A(u)\right) \prod_{k=1}^{i} L\left(v, s_{2 k}-s_{2 k-2}\right) z \|
\end{aligned}
$$




$$
\begin{aligned}
= & \sum_{i=1}^{m}\left(s_{2 i}-s_{2 i-2}\right) \| A(v) \prod_{k=1}^{i} L\left(v, s_{2 k}-s_{2 k-2}\right) z \\
& -A(u) \prod_{k=1}^{i} L\left(v, s_{2 k}-s_{2 k-2}\right) z \| \\
& <\sum_{i=1}^{m}\left(s_{2 i}-s_{2 i-2}\right) \cdot \varepsilon \\
= & x \cdot \varepsilon .
\end{aligned}
$$

Then, since $T(u, x) z=\Pi_{0}^{x} L(u, d I) z$ and $T(v, x) z=\Pi_{0}^{x} L(v, d I) z$ (see Remark 1.1), $\|T(u, x) z-T(v, x) z\| \leqq x \cdot \varepsilon$.

Proof of Part (1) of Theorem 1. Let $p \in D$, let $a, b \geqq 0$, and let $\varepsilon>0$. Let $M=\left\{\prod_{i=1}^{m} T\left(r_{2 i-1},\left|r_{2 i}-r_{2 i-2}\right|\right) p \mid x \in[a, b]\right.$ and $\left\{r_{i}\right\}_{i=0}^{2 m}$ is a chain from $a$ to $x$. Then $M$ is a bounded subset of $D$ by Lemma 1.1. There exists $\delta>0$ such that if $u, v \in[a, b],|u-v|<\delta, 0 \leqq x \leqq 1$ and $z \in M$, then $\|T(u, x) z-T(v, x) z\| \leqq \varepsilon \cdot x$. Let $\left\{s_{i}\right\}_{i=0}^{2 m}$ be a chain from $a$ to $b$ with norm $<\min \{\delta, 1\}$ and let $\left\{t_{i}\right\}_{i=0}^{2 n}$ be a refinement of $\left\{s_{i}\right\}_{i=0}^{2 m}$, i.e., there is an increasing sequence $u$ such that $u_{0}=0, u_{m}=n$, and if $1 \leqq i \leqq m s_{2 i}=t_{2 u_{i}}$. For $1 \leqq i \leqq m$ let $K_{i}=T\left(s_{2 i-1},\left|s_{2 i}-s_{2 i-2}\right|\right)$ and let $J_{i}=\prod_{j=u_{i-1}+1}^{u_{i}} T\left(t_{2 j-1},\left|t_{2 j}-t_{2 j-2}\right|\right)$. Then,

$$
\begin{aligned}
&\left\|\prod_{i=1}^{m} T\left(t_{2 i-1},\left|t_{2 i}-t_{2 i-2}\right|\right) p-\prod_{i=1}^{m} T\left(s_{2 i-1},\left|s_{2 i}-s_{2 i-2}\right|\right) p\right\| \\
&=\left\|\prod_{i=1}^{n} J_{i} p-\prod_{i=1}^{m} K_{i} p\right\| \\
& \leqq \sum_{i=1}^{m}\left\|\prod_{j=i}^{m} J_{j} \prod_{k=1}^{i-1} K_{k} p-\prod_{j=i+1}^{m} J_{j} \prod_{k=1}^{i} K_{k} p\right\| \\
& \leqq \sum_{i=1}^{m}\left\|J_{i} \prod_{k=1}^{i-1} K_{k} p-K_{i} \prod_{k=1}^{i-1} K_{k} p\right\| \\
&= \sum_{i=1}^{m} \| \prod_{j=u_{i-1}+1}^{u_{i}} T\left(t_{2 j-1},\left|t_{2 j}-t_{2 j-2}\right|\right) \prod_{k=1}^{i-1} K_{k} p \\
&-\prod_{j=u_{i-1}+1}^{u_{i}} T\left(s_{2 i-1},\left|t_{2 j}-t_{2 j-2}\right|\right) \prod_{k=1}^{i-1} K_{k} p \| \\
& \leqq \sum_{i=1}^{m} \sum_{j=u_{i-1}+1}^{u_{i}} \| \prod_{r=j}^{u_{i}} T\left(s_{2 i-1},\left|t_{2 r}-t_{2 r-2}\right|\right) \prod_{h=u_{i-1}+1}^{j-1} T\left(t_{2 h-1},\left|t_{2 h}-t_{2 h-2}\right|\right) \prod_{k=1}^{i-1} K_{k} p \\
&-\prod_{r=j+1}^{u_{i}} T\left(s_{2 i-1},\left|t_{2 r}-t_{2 r-2}\right|\right) \prod_{h=u_{i-1}+1}^{j} T\left(t_{2 h-1},\left|t_{2 h}-t_{2 h-2}\right|\right) \prod_{k=1}^{i-1} K_{k} p \| \\
& \leqq \sum_{i=1}^{m} \sum_{j=u_{i-1}+1}^{u_{i}} \| T\left(s_{2 i-1},\left|t_{2 j}-t_{2 j-2}\right|\right) \prod_{h=u_{i-1}+1}^{j-1} T\left(t_{2 h-1},\left|t_{2 h}-t_{2 h-2}\right|\right) \prod_{k=1}^{i-1} K_{k} p \\
&-T_{k=1} T\left(t_{2 j-1},\left|t_{2 j}-t_{2 j-2}\right|\right) \prod_{h=u_{i-1}+1}^{j-1} T\left(t_{2 h-1},\left|t_{2 h}-t_{2 h-2}\right|\right) \prod_{k=1}^{i-1} K_{k} p \| \\
& \leqq \sum_{i=1}^{m} \sum_{j=u_{i-1}+1}^{u_{i}}\left|t_{2 j}-t_{2 j-2}\right| \cdot \varepsilon=|b-a| \cdot \varepsilon \cdot
\end{aligned}
$$

Hence, $\prod_{a}^{b} T(I, d I) p$ exists. Further, using the fact that $D$ is dense 
in $S$ and $T(u, x)$ is nonexpansive for $u, x \geqq 0$ one sees that if $p \in S$, $a, b \geqq 0$, then $\Pi_{a}^{b} T(I, d I) p$ exists and thus part (1) of Theorem 1 is proved.

Before proving part (2) of Theorem 1 three lemmas will be proved each under the hypothesis of Theorem 1.

Lemma 1.4. If $p, q \in S, a, c \geqq 0$, and $b \in[a, c]$, then the following are true:

( i ) $\left\|\prod_{a}^{b} T(I, d I) p-\prod_{a}^{b} T(I, d I) q\right\| \leqq\|p-q\|$.

(ii ) $\prod_{b}^{c} T(I, d I) \prod_{a}^{b} T(I, d I) p=\prod_{a}^{c} T(I, d I) p$.

(iii) If $p \in D$ then $\left\|\Pi_{a}^{b} T(I, d I) p-p\right\| \leqq|b-a| \cdot \max _{u \in[a, b]}\|A(u) p\|$.

Proof. Parts (i) and (ii) follow from the nonexpansive property of $T(u, x), u, x \geqq 0$. Part (iii) follows from Lemma 1.1 .

LemmA 1.5. If $M$ is a bounded subset of $D, a, b \geqq 0$, and $\varepsilon>0$, there exists $\delta>0$ such that if $u, v \in[a, b],|v-u|<\delta, w \in[u, v]$, and $z \in M$, then

$$
\left\|\Pi_{u}^{v} T(I, d I) z-T(w,|v-u|) z\right\| \leqq|v-u| \cdot \varepsilon .
$$

Proof. Let $M^{\prime}=\left\{\prod_{i=1}^{m} T\left(s_{2 i-1},\left|s_{2 i}-s_{2 i-2}\right|\right) z \mid z \in M, x, y \in[a, b],\left\{s_{i}\right\}_{2 \times 0}^{2 m}\right.$ is a chain from $y$ to $x$. Then $M^{\prime}$ is a bounded subset of $D$ by Lemma 1.1. By Lemma 1.3 there exists $\delta>0$ such that if $u, v \in[a, b],|u-v|<$ $\delta, z \in M^{\prime}$ and $0 \leqq x \leqq 1$, then $\|T(u, x) z-T(v, x) z\| \leqq x \cdot \varepsilon$. Let $u, v \in[a, b],|v-u|<\min \{\delta, 1\}, w \in[u, v], z \in M$, and let $\left\{s_{i}\right\}_{i=0}^{2 m}$ be a chain from $u$ to $v$. Then,

$$
\begin{array}{rl}
\| \prod_{i=1}^{m} & T\left(s_{2 i-1},\left|s_{2 i}-s_{2 i-2}\right|\right) z-T(w,|v-u|) z \| \\
= & \left\|\prod_{i=1}^{m} T\left(s_{2 i-1},\left|s_{2 i}-s_{2 i-2}\right|\right) z-\prod_{2=1}^{m} T\left(w,\left|s_{2 i}-s_{2 i-2}\right|\right) z\right\| \\
\leqq & \sum_{i=1}^{m} \| T\left(s_{2 i-1},\left|s_{2 i}-s_{2 i-2}\right|\right) \prod_{j=1}^{i-1} T\left(s_{2 j-1},\left|s_{2 j}-s_{2 j-2}\right|\right) z \\
& \quad-T\left(w,\left|s_{2 i}-s_{2 i-2}\right|\right) \prod_{j=1}^{i-1} T\left(s_{2 j-1},\left|s_{2 j}-s_{j-2}\right|\right) z \| \\
\leqq & \sum_{i=1}^{m}\left|s_{2 i}-s_{2 i-2}\right| \cdot \varepsilon \\
= & |v-u| \cdot \varepsilon .
\end{array}
$$

Thus, $\| \Pi_{u}^{v} T(I, d I) z-T(w,|v-u|) z|| \leqq|v-u| \cdot \varepsilon$.

LEMmA 1.6. If $M$ is a bounded subset of $D, a, b \geqq 0$, and $\varepsilon>0$, there exists $\delta>0$ such that if $u, v \in[a, b], w \in[u, v],|v-u|<\delta, z \in M$, 
and $\left\{s_{i}\right\}_{i=0}^{2 m}$ is a chain from $u$ to $v$, then

$$
\left\|\prod_{i=1}^{m} L\left(s_{2 i-1},\left|s_{2 i}-s_{2 i-2}\right|\right) z-\prod_{i=1}^{m} L\left(w,\left|s_{2 i}-s_{2 i-2}\right|\right) z\right\| \leqq|v-u| \cdot \varepsilon .
$$

Proof. An argument similar to the one in Lemma 1.3 proves Lemma 1.6.

Proof of Part (2) of Theorem 1. Let $p \in D, a, b \geqq 0$, and $\varepsilon>0$. Let $M=\left\{\prod_{a}^{x} T(I, d I) p \mid x \in[a, b]\right\}$. Then $M$ is a bounded subset of $D$ by Lemma 1.4. By Lemmas 1.5 and 1.6 there exists $\delta>0$ such that if $u, v \in[a, b], w \in[u, v],|u-v|<\delta, z \in M$, and $\left\{s_{i}\right\}_{i=0}^{2 m}$ is a chain from $u$ to $v$, then

$$
\left\|\prod_{i=1}^{m} L\left(s_{2 i-1},\left|s_{2 i}-s_{2 i-2}\right|\right) z-\prod_{i=1}^{m} L\left(w,\left|s_{2 i}-s_{2 i-2}\right|\right) z\right\| \leqq|v-u| \cdot \varepsilon / 3|b-a|
$$

and $\left\|\Pi_{u}^{v} T(I, d I) z-T(w,|v-u|) z\right\| \leqq|v-u| \cdot \varepsilon / 3|b-a| . \quad$ Let $\left\{r_{i}\right\}_{i=0}^{2 q}$ be a chain from $a$ to $b$ with norm $<\delta$. Let $\left\{s_{i}\right\}_{i=0}^{2 m}$ be a refinement of $\left\{r_{i}\right\}_{i=0}^{2 q}$ such that there exists an increasing sequence $u$ such that $u_{0}=0, u_{q}=m$, if $1 \leqq i \leqq q r_{2 i}=s_{2 u_{i}}$, and if $1 \leqq i \leqq q$ and $\left\{t_{k}\right\}_{k=0}^{2 n}$ is a refinement of $\left\{s_{j}\right\}_{j=2 u_{i-1}}^{2 u_{i}}$, then

$$
\begin{aligned}
& \| \prod_{k=1}^{n} L\left(r_{2 i-1},\left|t_{2 k}-t_{2 k-2}\right|\right) \prod_{a}^{r_{2 i-2}} T(I, d I) p \\
& \quad-T\left(r_{2 i-1},\left|r_{2 i}-r_{2 i-2}\right|\right) \prod_{a}^{r_{2 i-2}} T(I, d I) p \| \leqq\left|r_{2 i}-r_{2 i-2}\right| \cdot \varepsilon / 3|b-a| .
\end{aligned}
$$

(Note that if

$$
\begin{aligned}
1 & \leqq i \leqq q T\left(r_{2 i-1},\left|r_{2 i}-r_{2 i-2}\right|\right) \prod_{a}^{r_{2 i-2}} T(I, d I) p \\
& =\prod_{r_{2 i-2}}^{r_{2 i}} L\left(r_{2 i-1}, d I\right) \prod_{a}^{r_{2 i-2}} T(I, d I) p=\prod_{r_{2 i}}^{r_{2 i-2}} L\left(r_{2 i-1}, d I\right) \prod_{a}^{r_{2 i-2}} T(I, d I) p
\end{aligned}
$$

- see Remark 1.1). Let $\left\{t_{i}\right\}_{i=0}^{2 n}$ be a refinement of $\left\{s_{i}\right\}_{i=0}^{2 m}$ and let $v$ be an increasing sequence such that $v_{0}=0, v_{m}=n$, and if $1 \leqq i \leqq m$ $s_{2 i}=t_{2 v_{i}}$. Then,

$$
\begin{array}{rl}
\| \prod_{i=1}^{n} & L\left(t_{2 i-1},\left|t_{2 i}-t_{2 i-2}\right|\right) p-\prod_{a}^{b} T(I, d I) p \| \\
= & \| \prod_{i=1}^{q} \prod_{j=u_{i-1}+1}^{u_{i}} \prod_{k=v_{j-1}+1}^{v_{j}} L\left(t_{2 k-1},\left|t_{2 k}-t_{2 k-2}\right|\right) p \\
& -\prod_{i=1}^{q} \prod_{r_{2 i-2}}^{r_{2 i}} T(I, d I) p \| \\
\leqq & \sum_{i=1}^{q} \| \prod_{j=u_{i-1}+1}^{u_{i}} \prod_{k=v_{j-1}+1}^{v_{j}} L\left(t_{2 k-1},\left|t_{2 k}-t_{2 k-2}\right|\right) \prod_{a}^{r_{2 i-2}} T(I, d I) p \\
& \quad-\prod_{r_{2 i-2}}^{r_{2 i}} T(I, d I) \prod_{a}^{r_{2 i-2}} T(I, d I) p \|
\end{array}
$$




$$
\begin{aligned}
\leqq & \sum_{i=1}^{q}\left|r_{2 i}-r_{2 i-2}\right| \cdot \varepsilon / 3|b-a| \\
& +\sum_{i=1}^{q}||_{j=u_{i-1}+1} \prod_{k=v_{j-1}+1}^{u_{i}} L\left(r_{2 i-1},\left|t_{2 k}-t_{2 k-2}\right|\right) \prod_{a}^{v_{2 i-2}} T(I, d I) p \\
& -T\left(r_{2 i-1},\left|r_{2 i}-r_{2 i-2}\right|\right) \prod_{a}^{r_{2 i-2}} T(I, d I) p|| \\
& +\sum_{i=1}^{q}\left|r_{2 i}-r_{2 i-2}\right| \cdot \varepsilon / 3|b-a| \\
\leqq &
\end{aligned}
$$

Thus, $\prod_{a}^{b} L(I, d I) p$ exists and is $\prod_{a}^{b} T(I, d I) p$ for $p \in D$. Further, using the fact that $D$ is dense in $S$ and $L(u, x)$ is nonexpansive for $u, x \geqq 0$ one sees that $\prod_{a}^{b} L(I, d I) p=\prod_{a}^{b} T(I, d I) p$ for all $p \in S$.

Define $U(b, a) p=\prod_{a}^{b} T(I, d I) p$ for $p \in S$ and $a, b \geqq 0$.

Proof of Theorem 2. Parts (1), (2), and (3) of Theorem 2 follow from Lemma 1.4. Suppose that $p \in S, 0 \leqq a \leqq t$, and $U(t, a) p \in D$. Let $\varepsilon>0$. There exists $\delta_{1}>0$ such that if $0<h<\delta_{1}$

$$
\|A(t) T(t, h) U(t, a) p-A(t) U(t, a) p\|<\varepsilon / 2
$$

(see Definition 1.1, part (4)). By Lemma 1.5 there exists $\delta_{2}>0$ such that if $0<h<\delta_{2}\|U(t+h, t) U(t, a) p-T(t, h) U(t, a) p\|<h \cdot \varepsilon / 2$. Then, if $0<h<\min \left\{\delta_{1}, \delta_{2}\right\}$,

$$
\begin{aligned}
& \|(1 / h)(U(t+h, a) p-U(t, a) p)-A(t) U(t, a) p\| \\
& =\|(1 / h)(U(t+h, t) U(t, a) p-U(t, a) p)-A(t) U(t, a) p\| \\
& \quad<\varepsilon / 2+\|(1 / h)(T(t, h) U(t, a) p-U(t, a) p)-A(t) U(t, a) p\| \\
& =\varepsilon / 2+\left\|1 / h \int_{0}^{h}[A(t) T(t, u) U(t, a) p-A(t) U(t, a) p] d u\right\|<\varepsilon
\end{aligned}
$$

Hence, $\partial^{+} U(t, a) p / \partial t=A(t) U(t, a) p$. Suppose that $p \in S, 0<s \leqq b$, and $U(s, b) p \in D$. Let $\varepsilon>0$. There exists $\delta_{1}>0$ such that if $0<h<\delta_{1}$ then $0 \leqq s-h$ and $\|A(s) T(s, h) U(s, b) p-A(s) U(s, b) p\|<\varepsilon / 2$. By Lemma 1.5 there exists $\delta_{2}>0$ such that if $0<h<\delta_{2}$

$$
\|U(s-h, s) U(s, b) p-T(s, h) U(s, b) p\|<h \cdot \varepsilon / 2 .
$$

Then, if $0<h<\min \left\{\delta_{1}, \delta_{2}\right\}$

$$
\begin{aligned}
&\|(1 /-h)(U(s-h, b) p-U(s, b) p)-(-A(s) U(s, b) p)\| \\
&=\|(1 / h)(U(s-h, s) U(s, b) p-U(s, b) p)-A(s) U(s, b) p\| \\
&<\varepsilon / 2+\|(1 / h)(T(s, h) U(s, b) p-U(s, b) p)-A(s) U(s, b) p\| \\
&= \varepsilon / 2+\left\|1 / h \int_{0}^{h}[A(s) T(s, u) U(s, b) p-A(s) U(s, b) p] d u\right\|<\varepsilon .
\end{aligned}
$$

Hence, $\partial^{-} U(s, b) p / \partial s=-A(s) U(s, b) p$. 
3. Product integral representation in the uniform case. For Theorem $3 A$ is required to satisfy, in addition to conditions (I), (II), (III) of $\S 1$, the following:

(IV) For each $t \geqq 0 A(t)$ has domain all of $S$.

(V) If $0 \leqq a \leqq b, M$ is a bounded subset of $S$, and $\varepsilon>0$, there exists $\delta>0$ such that if $u \in[a, b], z, w \in M$, and $\|z-w\|<\delta$, then

$$
\|A(u) z-A(u) w\|<\varepsilon \text {. }
$$

Theorem 3. Let A satisfy conditions (I)-(V) and define

$$
M(u, v)=(I+v A(u))
$$

for $u, v \geqq 0$. If $p \in S$ and $a, b \geqq 0$, then $\prod_{a}^{b} M(I, d I) p=U(b, a) p$.

Before proving Theorem 3, three lemmas will be proved each under the hypothesis of Theorem 3.

Lemma 3.1. Let $p \in S$ and let $a, b \geqq 0$. There is a neighborhood $N_{p, o}$ about $p$, a positive number $\gamma$, and a positive number $K$ such that if $q \in N_{p, \delta}, x, y \in[a, b],|y-x|<\gamma$, and $\left\{s_{i}\right\}_{i=0}^{2 m}$ is a chain from $x$ to $y$, then

$$
\left\|\prod_{i=1}^{m} M\left(s_{2 i-1},\left|s_{2 i}-s_{2 i-2}\right|\right) q-q\right\| \leqq|y-x| \cdot K .
$$

Proof. There exists a positive number $K$ such that if $u \in[a, b]$ and $q \in N_{p, 1}$ then $\|A(u) q\| \leqq K$. Let $\delta=1 / 2$ and let $\gamma=1 / 2 K$. Let $q \in N_{p, o}, x, y \in[a, b],|y-x|<\gamma,\left\{s_{i}\right\}_{i=0}^{2 m}$ a chain from $x$ to $y, 1 \leqq j \leqq m-$ 1 , and suppose that $\left\|\prod_{i=1}^{j} M\left(s_{2 i-1},\left|s_{2 i}-s_{2 i-2}\right|\right) q-q\right\| \leqq\left|s_{2 j}-s_{0}\right| \cdot K$. Then, $\prod_{i=1}^{j} M\left(s_{2 i-1},\left|s_{2 i}-s_{2 i-2}\right|\right) q \in N_{p, 1}$ and so

$$
\begin{aligned}
& \left\|\prod_{i=1}^{j+1} M\left(s_{2 i-1},\left|s_{2 i}-s_{2 i-2}\right|\right) q-q\right\| \\
& \leqq \\
& \quad\left\|\prod_{i=1}^{j} M\left(s_{2 i-1},\left|s_{2 i}-s_{2 i-2}\right|\right) q-q\right\| \\
& \quad+\left|s_{2 j+2}-s_{2 j}\right| \cdot\left\|A\left(s_{2 j+1}\right) \prod_{i=1}^{j} M\left(s_{2 i-1},\left|s_{2 i}-s_{2 i-2}\right|\right) q\right\| \\
& \leqq \\
& \quad\left|s_{2 j+2}-s_{0}\right| \cdot K .
\end{aligned}
$$

Lemma 3.2. If $p \in S$ and $a \geqq 0$ then $U(t, a) p$ is continuous in $t$.

Proof. Let $p \in S$ and $a, b \geqq 0$. In a manner similar to Lemma 3.1 one proves the following: There is a neighborhood $N_{q, \delta}$ about $q=$ $U(b, a) p, \gamma>0$, and $K>0$ such that if $z \in N_{q, \hat{o}}, x, y \in[a, b],|y-x|<\gamma$, and $\left\{s_{i}\right\}_{i=0}^{2 m}$ is a chain from $x$ to $y$ then 


$$
\left\|\prod_{m}^{i=1}\left(I-\left|s_{2 i}-s_{2 i-2}\right| A\left(s_{2 i-1}\right)\right) z-z\right\| \leqq|y-x| \cdot K .
$$

Let $\varepsilon>0$, let $x \in[a, b]$ such that $|x-b|<\gamma$, let $\left\{s_{i}\right\}_{i=0}^{2 m}$ be a chain from $a$ to $b$ and $k \leqq m$ an integer such that $s_{2 k}=x$ and

$$
\left\|U(b, a) p-\prod_{i=1}^{m} L\left(s_{2 i-1},\left|s_{2 i}-s_{2 i-2}\right|\right) p\right\|<\min \{\varepsilon, \delta\}
$$

and

$$
\left\|U(x, a) p-\prod_{i=1}^{k} L\left(s_{2 i-1},\left|s_{2 i}-s_{2 i-2}\right|\right) p\right\|<\varepsilon
$$

Then,

$$
\begin{aligned}
& \|U(x, a) p-U(b, a) p\| \\
& \quad<2 \varepsilon+\| \prod_{m}^{i=k+1}\left(I-\left|s_{2 i}-s_{2 i-2}\right| A\left(s_{2 i-1}\right)\right) \prod_{i=1}^{m} L\left(s_{2 i-1},\left|s_{2 i}-s_{2 i-2}\right|\right) p \\
& \quad-\prod_{i=1}^{m} L\left(s_{2 i-1},\left|s_{2 i}-s_{2 i-2}\right|\right) p \| \\
& \quad<2 \varepsilon+|b-x| \cdot K .
\end{aligned}
$$

Then, $\lim _{x \rightarrow b} U(x, a) p=U(b, a) p$ for $x \in[a, b]$. Further, by Lemma 1.4 $\lim _{x \rightarrow b} U(x, a) p=U(b, a) p$ for $x \notin[a, b]$.

LEммA 3.3. Let $p \in S$ and $a \geqq 0$. There exists a neighborhood $N_{p, \dot{o}}$ about $p$ and $\gamma>0$ such that the following are true:

(1) If $\varepsilon>0$ there exists $\alpha>0$ such that if $q \in N_{p, i}, a \leqq x \leqq a+\gamma$, and $\left\{s_{i}\right\}_{i-0}^{2 m}$ is a chain from a to $x$ with norm $<\alpha$, then

$$
\left\|\prod_{i=1}^{m} M\left(s_{2 i-1}, s_{2 i}-s_{2 i-2}\right) q-U(x, a) q\right\|<\varepsilon .
$$

and

(2) If $\varepsilon>0$ there exists $\alpha>0$ such that if $q \in N_{p, \delta}, \max \{0, a-\gamma\} \leqq$ $x \leqq a$, and $\left\{s_{i}\right\}_{i=0}^{2 m}$ is a chain from $a$ to $x$ with norm $<\alpha$, then

$$
\left\|\prod_{i=1}^{m} M\left(s_{2 i-1},\left|s_{2 i}-s_{2 i-2}\right|\right) q-U(x, a) q\right\|<\varepsilon \text {. }
$$

Proof. By Lemma 3.1 there exists $\delta>0$ and $\gamma>0$ such that if $q \in N_{p, \delta}, a \leqq x \leqq a+\gamma$, and $\left\{s_{i}\right\}_{i=0}^{2 m}$ is a chain from $a$ to $x$ then

$$
\prod_{i=1}^{m} M\left(s_{2 i-1}, s_{2 i}-s_{2 i-2}\right) q \in N_{p, 2 \delta}
$$

Let $\varepsilon>0$. By Lemma 1.5 there exists $\alpha_{1}>0$ such that if

$$
u, v \in[a, a+\gamma], 0 \leqq v-u<\alpha_{1}, u \leqq w \leqq v,
$$


and $q \in N_{p, 2 i}$, then $\|U(v, u) q-T(w, v-u) q\| \leqq(v-u) \cdot \varepsilon / 2 \gamma$. There exists $\alpha_{2}>0$ such that if $q \in N_{p, 2 \delta}, u \in[a, a+\gamma]$, and $0 \leqq x<\alpha_{2}$, then $\|A(u) T(u, x) q-A(u) q\|<\varepsilon / 2 \gamma$ (Note that

$$
\begin{aligned}
& \|T(u, x) q-q\|=\left\|\int_{0}^{x} A(u) T(u, t) q d t\right\| \leqq x \cdot\|A(u) q\| \leqq x . \\
& \left.\quad \times\left(\max \|A(t) z\|, t \in[a, a+\gamma], z \in N_{p, 2 \delta}\right)\right) .
\end{aligned}
$$

Let $\alpha=\min \left\{\alpha_{1}, \alpha_{2}\right\}$, let $q \in N_{p, \hat{\alpha}}$, let $a \leqq x \leqq \alpha+\gamma$, and let $\left\{s_{i}\right\}_{i=10}^{2 m}$ be a chain from $a$ to $x$ with norm $<\alpha$. Then,

$$
\begin{array}{rl}
\| \prod_{i=1}^{m} & M\left(s_{2 i-1}, s_{2 i}-s_{2 i-2}\right) q-U(x, a) q \| \\
= & \left\|\prod_{i=1}^{m} M\left(s_{2 i-1}, s_{2 i}-s_{2 i-2}\right) q-\prod_{i=1}^{m} U\left(s_{2 i}, s_{2 i-2}\right) q\right\| \\
\leqq & \sum_{i=1}^{m} \| U\left(s_{2 i}, s_{2 i-2}\right) \prod_{j=1}^{i-1} M\left(s_{2 j-1}, s_{2 j}-s_{2 j-2}\right) q \\
& \quad-M\left(s_{2 i-1}, s_{2 i}-s_{2 i-2}\right) \prod_{j=1}^{i-1} M\left(s_{2 j-1}, s_{2 j}-s_{2 j-2}\right) q \| \\
& <\varepsilon / 2+\sum_{i=1}^{m} \| T\left(s_{2 i-1}, s_{2 i}-s_{2 i-2}\right) \prod_{j=1}^{i-1} M\left(s_{2 j-1}, s_{2 j}-s_{2 j-2}\right) q \\
& \quad-M\left(s_{2 i-1}, s_{2 i}-s_{2 i-2}\right) \prod_{j=1}^{i-1} M\left(s_{2 j-1}, s_{2 j}-s_{2 j-2}\right) q \| \\
= & \varepsilon / 2+\sum_{i=1}^{m} \| \int_{0}^{s_{2 i-s_{2 i-2}}}\left[A\left(s_{2 i-1}\right) T\left(s_{2 i-1}, t\right) \prod_{j=1}^{i-1} M\left(s_{2 j-1}, s_{2 j}-s_{2 j-2}\right) q\right. \\
& \left.\quad-A\left(s_{2 i-1}\right) \prod_{j=1}^{i-1} M\left(s_{2 j-1}, s_{2 j}-s_{2 j-2}\right) q\right] d t \| \\
< & \varepsilon / 2+\sum_{i=1}^{m}\left(s_{2 i}-s_{2 i-2}\right) \cdot \varepsilon / 2 \gamma<\varepsilon .
\end{array}
$$

A similar argument proves part (2) of the lemma.

Proof of Theorem 3. Let $p \in S$ and $0 \leqq a<b$. Suppose that if $a \leqq x<b \prod_{a}^{x} M(I, d I) p$ exists and is $U(x, a) p$. Let $a \leqq x<b$, let $\left\{s_{i}\right\}_{i=0}^{2 m}$ be a chain from $a$ to $b$, and let $j<m$ such that $s_{2 j}=x$. One uses the inequality

$$
\begin{aligned}
& \left\|U(b, a) p-\prod_{i=1}^{m} M\left(s_{2 i-1}, s_{2 i}-s_{2 i-2}\right) p\right\| \\
& \leqq \\
& \quad+U(b, a) p-\prod_{a}^{x} M(I, d I) p \| \\
& \quad+\left\|\prod_{a}^{x} M(I, d I) p-\prod_{i=1}^{j} M\left(s_{2 i-1}, s_{2 i}-s_{2 i-2}\right) p\right\| \\
& \quad+\| \prod_{i=1}^{j} M\left(s_{2 i-1}, s_{2 i}-s_{2 i-2}\right) p \\
& \quad-\prod_{i=j+1}^{m} M\left(s_{2 i-1}, s_{2 i}-s_{2 i-2}\right) \prod_{i=1}^{j} M\left(s_{2 i-1}, s_{2 i}-s_{2 i-2}\right) p \|
\end{aligned}
$$


and Lemmas 3.1 and 3.2 to show $\prod_{a}^{b} M(I, d I) p$ exists and is $U(b, a) p$. Suppose now that for $a \leqq x \leqq b \prod_{a}^{x} M(I, d I) p=U(x, a) p$. Let $b<x$, let $\left\{s_{i}\right\}_{i=0}^{2 m}$ be a chain from $a$ to $x$, and let $j<m$ such that $s_{2 j}=b$. One uses the inequality

$$
\begin{aligned}
& \left\|U(x, a) p-\prod_{i=1}^{m} M\left(s_{2 i-1}, s_{2 i}-s_{2 i-2}\right) p\right\| \\
& \leqq \\
& \quad+\left\|U(x, b) U(b, a) p-U(x, b) \prod_{i=1}^{j} M\left(s_{2 i-1}, s_{2 i}-s_{2 i-2}\right) p\right\| \\
& \quad-\prod_{i=j+1}^{m} M\left(s_{2 i-1}, s_{2 i}-s_{2 i-2}\right) \prod_{i=1}^{j} M\left(s_{2 i-1}, s_{2 i}-s_{2 i-2}\right) p \|
\end{aligned}
$$

and Lemma 3.3 to show that there exists $\gamma>0$ such that if $b \leqq x<b+\gamma$ then $\prod_{a}^{x} M(I, d I) p$ exists and is $U(x, a) p$. Thus, if $p \in S$ and $0 \leqq a \leqq b$ then $\prod_{a}^{b} M(I, d I) p$ exists and is $U(b, a) p$. With a similar argument one shows that for $p \in S$ and $0 \leqq a \leqq b \prod_{b}^{a} M(I, d I) p$ exists and is $U(a, b) p$.

4. Examples. In conclusion two examples will be given.

ExAmple 1. Let $S$ be the Hilbert space and let $A$ be densely defined and $m$-monotone on $S$ (Definition 1.2). In M. Crandall and A. Pazy [2] and in T. Kato [6], it is shown that $B$ is the infinitesimal generator of a $\mathscr{C}$-semi-group on $S$ (Definition 1.1). Let $X$ be a function from $[0, \infty)$ to $S$ such that $X$ is continuous. Define $A(t) p=B p+X(t)$ for $p \in$ Domain $(B)$ and $t \geqq 0$. Then $A$ satisfies conditions (I)-(III).

EXAMPLE 2. Let $S$ be a Banach space and let $B$ be a mapping from $S$ to $S$ such that $B$ is $m$-monotone $S$ and uniformly continuous on bounded subsets of $S$. In [11] it is shown that $B$ is the infinitesimal generator of a $\mathscr{C}$-semi-group of mappings on $S$. Let $C$ be a continuous mapping from $[0, \infty)$ to $[0, \infty)$, let $D$ be a continuous mapping from $[0, \infty)$ to $(0, \infty)$, and let each of $E$ and $F$ be a continuous mapping from $[0, \infty)$ to $S$. Define $A(t) p=C(t) \cdot B(D(t) \cdot p+E(t))+F(t)$ for $t \geqq 0$ and $p \in S$. Suppose $t \geqq 0, \varepsilon>0$, and $p, q \in S$. Then,

$$
\begin{aligned}
&\|(I-\varepsilon A(t)) p-(I-\varepsilon A(t)) q\| \\
&=(1 / D(t)) \|(I-\varepsilon C(t) D(t) B)(D(t) p+E(t)) \\
&-(I-\varepsilon C(t) D(t) B)(D(t) q+E(t)) \| \\
& \geqq(1 / D(t))\|(D(t) p+E(t))-(D(t) q+E(t))\| \\
&=\|p-q\|
\end{aligned}
$$

and so $A(t)$ is monotone for $t \geqq 0$. Suppose $t \geqq 0, \varepsilon>0$, and $p \in S$. Let $q^{\prime}$ be in $S$ such that $(I-\varepsilon C(t) D(t) B) q^{\prime}=D(t) p+E(t)+\varepsilon D(t) F(t)$. 
Let $q=(1 / D(t))\left(q^{\prime}-E(t)\right)$. Then $(I-\varepsilon A(t)) q=p$ and so $A(t)$ is $m$ monotone. Then $A$ satisfies conditions (I)-(V).

\section{REFERENCES}

1. F. E. Browder, Nonlinear equations of evolution, Ann. of Math. 80 (1964), 485-523.

2. M. G. Crandall and A. Pazy, Nonlinear semi-groups of contractions and dissipative sets, J. Functional Analysis, 3 (1969), 376-418.

3. J. R. Dorroh, A class of nonlinear evolution equations in a Banach space (to appear) 4. E. Hille and R. S. Phillips, Functional analysis and semi-groups, rev. ed., Amer. Math. Soc. Coll. Pub., Vol. XXXI, 1957.

5. T. Kato, Integration of the equation of evolution in a Banach space, J. Math. Soc. Japan 5 (1958), 208-234.

6. - Nonlinear semi-groups and evolution equations, J. Math. Soc. Japan 19 (1967), 508-520.

7. Y. Kōmura, Nonlinear semi-groups in Hilbert space, J. Math. Soc. Japan 19 (1967), 493-507.

8. J. W. Neuberger, Product integral formulae for nonlinear expansive semi-groups and non-expansive evolution systems, J. Math. and Mech. (to appear)

9. P. E. Sobolevski, On equations of parabolic type in a Banach space, Trudy Moskov. Mat. Obšč. 10 (1961), 297-350.

10. G. F. Webb, Representation of nonlinear nonexpansive semi-groups of transformations in Banach space, J. Math. and Mech., 19 (1969), 159-170.

11. Nonlinear evolution equations and product integration in Banach spaces (to appear)

12. K. Yosida, Functional analysis, Springer Publishing Company, Berlin-HeidelbergNew York, 1965.

Received May 16, 1969.

VANDERBILT UNIVERSITY 



\section{PACIFIC JOURNAL OF MATHEMATICS}

\section{EDITORS}

\author{
H. SAMELSON \\ Stanford University \\ Stanford, California 94305 \\ Richard Pierce \\ University of Washington \\ Seattle, Washington 98105
}

J. DUGUNDJI

Department of Mathematics

University of Southern California

Los Angeles, California 90007

BASIL GORDON*

University of California

Los Angeles, California 90024

\section{ASSOCIATE EDITORS}
E. F. BECKENBACH
B. H. NeUmanN
F. WOLF
K. YoSHIDA

\section{SUPPORTING INSTITUTIONS}

UNIVERSITY OF BRITISH COLUMBIA CALIFORNIA INSTITUTE OF TECHNOLOGY

UNIVERSITY OF CALIFORNIA MONTANA STATE UNIVERSITY

UNIVERSITY OF NEVADA

NEW MEXICO STATE UNIVERSITY

OREGON STATE UNIVERSITY

UNIVERSITY OF OREGON

OSAKA UNIVERSITY

UNIVERSITY OF SOUTHERN CALIFORNIA
STANFORD UNIVERSITY

UNIVERSITY OF TOKYO

UNIVERSITY OF UTAH

WASHINGTON STATE UNIVERSITY

UNIVERSITY OF WASHINGTON

AMERICAN MATHEMATICAL SOCIETY CHEVRON RESEARCH CORPORATION TRW SYSTEMS

NAVAL WEAPONS CENTER 


\section{Pacific Journal of Mathematics}

\section{Vol. 32, No. $1 \quad$ January, 1970}

Robert Alexander Adams, Compact Sobolev imbeddings for unbounded domains ........................................ 1

Bernhard Amberg, Groups with maximum conditions .................. 9

Tom M. (Mike) Apostol, Möbius functions of order k............... 21

Stefan Bergman, On an initial value problem in the theory of two-dimensional transonic flow patterns ................... 29

Geoffrey David Downs Creede, Concerning semi-stratifiable spaces ...... 47

Edmond Dale Dixon, Matric polynomials which are higher

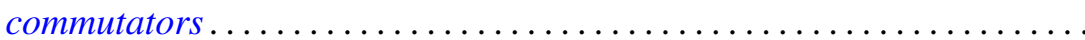

R. L. Duncan, Some continuity properties of the Schnirelmann density.

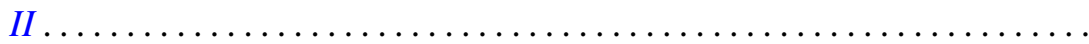

Peter Larkin Duren and Allen Lowell Shields, Coefficient multipliers of $H^{p}$

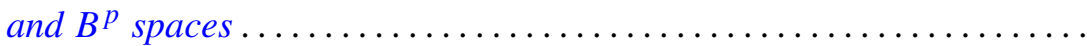

Hector O. Fattorini, On a class of differential equations for vector-valued

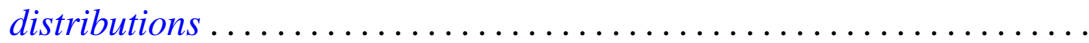

Charles Hallahan, Stability theorems for Lie algebras of derivations. . . . . . 105

Heinz Helfenstein, Local isometries of flat tori ................ 113

Gerald J. Janusz, Some remarks on Clifford's theorem and the Schur

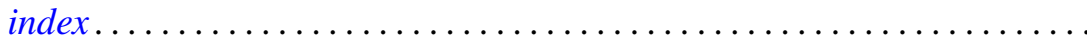

Joe W. Jenkins, Symmetry and nonsymmetry in the group algebras of discrete groups. ...

Herbert Frederick Kreimer, Jr., Outer Galois theory for separable

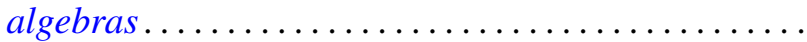

D. G. Larman and P. Mani, On visual hulls

R. Robert Laxton, On groups of linear recurrences. II. Elements of finite order.

Dong Hoon Lee, The adjoint group of Lie groups ...

James B. Lucke, Commutativity in locally compact rings

Charles Harris Scanlon, Rings of functions with certain Lipschitz

$$
\text { properties ............................... }
$$

Binyamin Schwarz, Totally positive differential systems .

James McLean Sloss, The bending of space curves into piecewise helical curves.

James D. Stafney, Analytic interpolation of certain multiplier spaces ...

Patrick Noble Stewart, Semi-simple radical classes.......

Hiroyuki Tachikawa, On left $\mathrm{QF}-3$ rings ...................... 255

Glenn Francis Webb, Product integral representation of time dependent nonlinear evolution equations in Banach spaces.... . . . 